\section{Infrared Spectroscopy of Some Mannans}

\section{Koji Kato, Masami Nitta and Takashi Mizuno}

\section{Department of Agricultural Chemistry, Faculty of Agriculture, Shizuoka University, Iwata-shi, Shizuoka-ken}

Received August 4, 1972

Infrared absorption spectra for hetero-mannans such as galacto-, for homo-mannans from yam (Dioscorea Batas Decne forma Icho ${ }^{4}$ ), alga (Halicoryne wrightii ${ }^{61}$ ) and yeast (Saccharomyces cerevisiae ${ }^{6}$ and Candida utilis $^{7}$ ) showed two characteristic absorption bands ${ }^{81}$ near 810 and 870 $\mathrm{cm}^{-1}$ (Fig. 1), which were not observed for other polysaccharides*; cellulose, xylan and galactan..$^{91}$

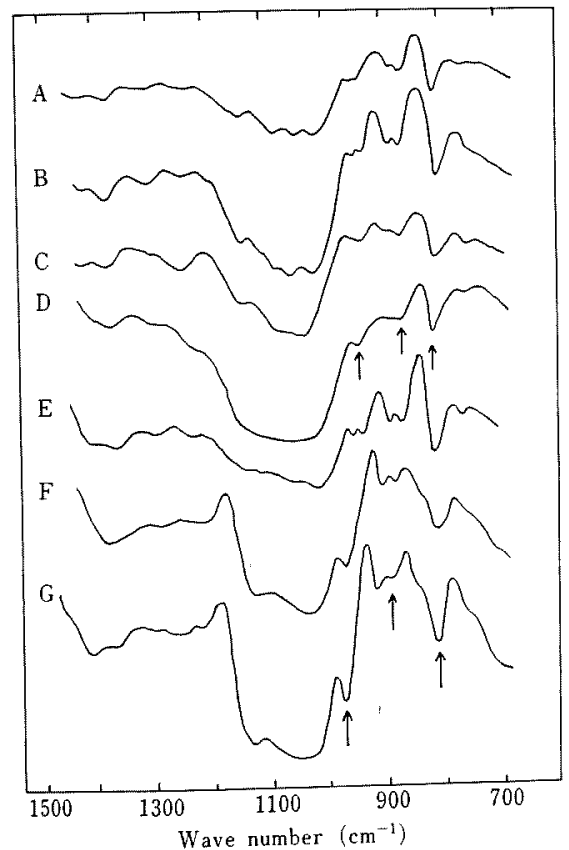

FIG. 1. IR-spectrum of the Mannan Obtained from

(A) Coffea arabica (Galactomannan) ${ }^{1 /}$

(B) Amorphophallus konjac (Glucomannan)2

(C) Prasiola japonica (Xylomannan) ${ }^{31}$

(D) Halicoryne wrightii (Mannan) ${ }^{4}$

(E) Dioscorea Batas Decne forma Icho (Mannan)

(F) Saccharomyces cerevisiae (Mannan) ${ }^{6}$

(G) Candida utilis (Mannan) ${ }^{7}$

* Inulin, $\beta-(2 \rightarrow 1)$ fructan, also shows the bands near 810 and $870 \mathrm{~cm}^{-1}$. However, they are not affected by acetylation.
Since these bands are also observed in the spectra of manno-oligosaccharides which can be regarded as the basic unit of the mannan (Figs. 3 and 4), it is reasonable to consider that they are caused by $\mathrm{D}$-mannose residue in the polymers irrespective of the configuration and position of the mannosidic linkage.

Absorption caused by ring-breathing frequency (type 3) of $\beta$-D-mannopyranose occurs at $770 \mathrm{~cm}^{-1}$, whereas that of methyl $\beta$-D-mannopyranoside occurs at $795 \mathrm{~cm}^{-1} \cdot{ }^{10}$ This will be suggestive that the bands near $810 \mathrm{~cm}^{-1}$ of the manno-oligosaccharides and mannans are restricted to type 3 absorption of Dmannose residue. In addition, it has been known that acetyl derivative of methyl $\beta$-D-mannopyranoside shows type 3 absorption at lower wave-number (747 $\mathrm{cm}^{-1}$ ) than that of original glycoside $\left(795 \mathrm{~cm}^{-1}\right) .{ }^{10}$ We observed the similar fact for epicellobiose; the sugar showed the band at $810 \mathrm{~cm}^{-1}$, while the acetate showed at $800 \mathrm{~cm}^{-1}$ Therefore, we may safely say that disappearance of the absorption band near $810 \mathrm{~cm}^{-1}$ and concurrent appearance of the band near 775 and $790 \mathrm{~cm}^{-1}$, on acetylation and on methylation of the mannan, respectively, are due to the shift of type 3 absorption band of D-mannose residue (Fig. 2).

It is interesting that the shift of type 3 to high wavenumber caused by glycosidation is in contrast to that of D-glucose to low wave-number; the absorption of $\alpha$-D-glucose series is observed at $774(\alpha$-D-glucose),

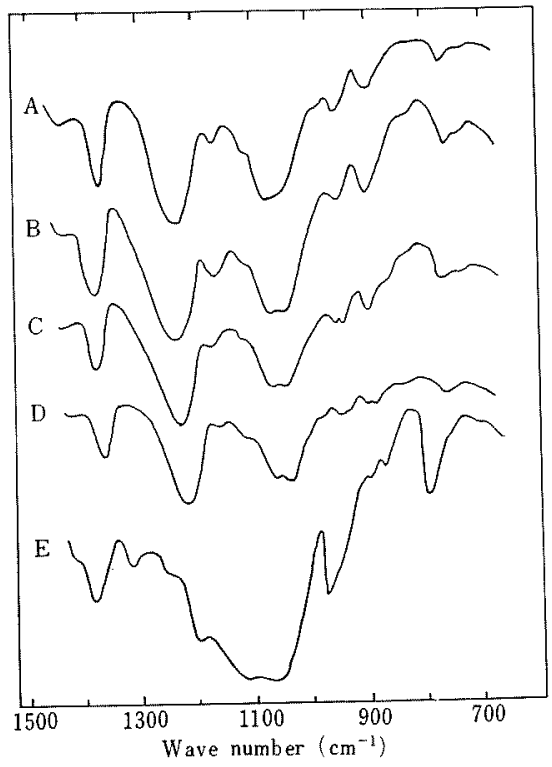

FIG. 2. IR-spectrum of the Mannan Derivative.

(A) Acetylated galactomannan (C. arabica)

(B) Acetylated glucomannan (A. konjac)

(C) Acetylated mannan ( $H$. wrightii)

(D) Acetylated xylomannan ( $P$. japonica)

(E) Methylated glucomannan (A. konjac) 


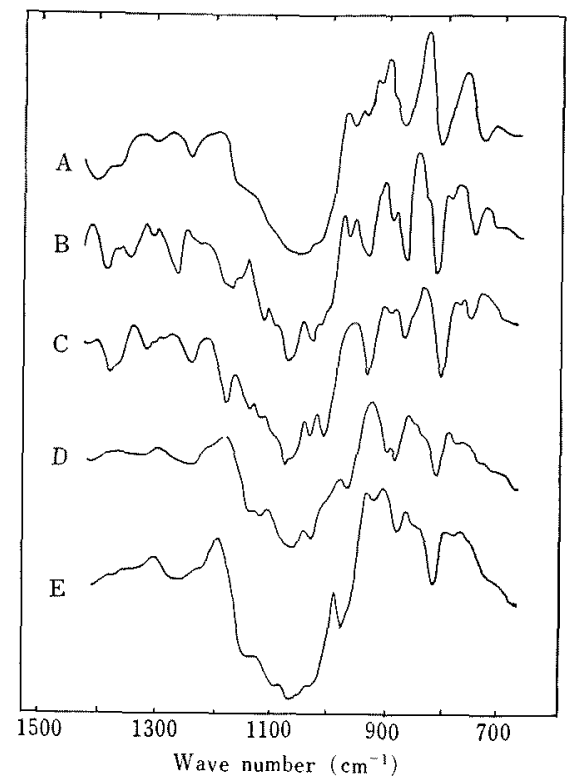

FIG. 3. IR-spectrum of the Manno-oligosaccharide. (A) $\beta-(1 \rightarrow 4)$ mannobiose $\quad$ (B) $\beta-(1 \rightarrow 4)$ mannotriose (C) $\beta-(1 \rightarrow 4)$ mannotetraose (D) $\beta-(1 \rightarrow 6)$ mannobiose (E) $\alpha-(1 \rightarrow 6)$ mannobiose

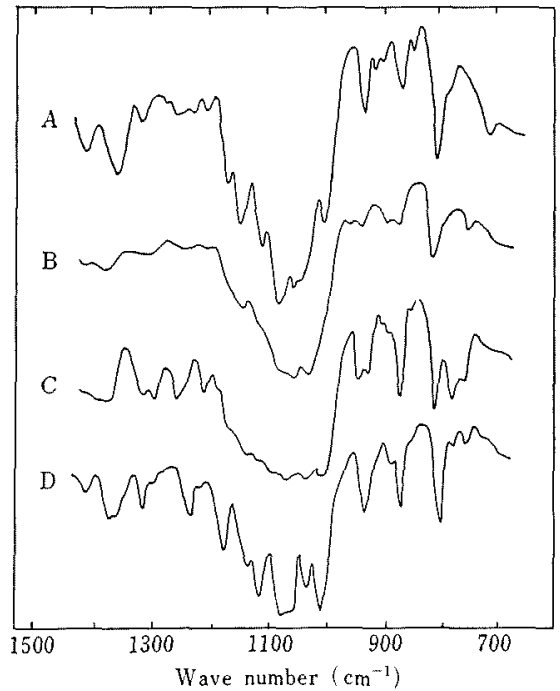

FIG. 4. IR-spectrum of the Manno-oligosaccharide Containing D-Glucose Residue at Reducing End.

(A) mannosyl glucose (B) mannobiosyl glucose (C) mannotriosyl glucose (D) mannotetraosyl glucose

745 (methyl $\alpha$-D-glucopyranoside) and $756 \mathrm{~cm}^{-1}$ (amylose), and in $\alpha$-D-glucose series, at 783 (methyl $\beta$-D-glucopyranoside), 773 (cellobiose) and $766 \mathrm{~cm}^{-1}$ (cellulose). ${ }^{10}$

The absorptions at 860 (for D-mannose) and $880 \mathrm{~cm}^{-1}$ (for the acetate) have been assigned to deformation of equatorial $\mathrm{C} 2-\mathrm{H}$ bond (type $2 \mathrm{c}$ ), ${ }^{101}$ which suggests that the band at $870 \mathrm{~cm}^{-1}$ observed in the manno. oligosaccharides and the mannans are also due to type $2 c$ absorption of D-mannose residue. But it is difficult to recognize type $2 c$ absorption of acetyl derivative, because of the presence of intensified type $2 \mathrm{~b}$ absorption in the near vicinity.

IR-spectrum of yam-mannan is clearly different from that of yeast-mannan in the region 940 to $980 \mathrm{~cm}^{-1}$ (Fig. 1). A band near $970 \mathrm{~cm}^{-1}$ was observed for not only yeast-mannan but also for manno-oligosaccharides except mannotetraose and the oligosaccharides containing $D$-glucose residue at the reducing end. This suggests that the bands near $970 \mathrm{~cm}^{-1}$ of the oligosaccharides and of yeast-mannan are different in assignment, respectively. On the other hand, a band near $940 \mathrm{~cm}^{-1}$ was observed for yam-mannan and for all of the manno-oligosaccharides except $\alpha$ - and $\beta$ $(1 \rightarrow 6)$ mannobioses. From these facts, it would be claimed that the absorptions, near 940 and $970 \mathrm{~cm}^{-1}$, are valuable for recognition of the main glycosidic linkage of the mannan.

\section{EXPERIMENTAL}

Spectra of the sugars in $\mathrm{KBr}$ disk were recorded with a Japan Spectroscopic Co., Ltd., Model IR-S infrared spectrometer. The mannans used were extracted with water (or alkali) and purified via the coppercomplex. $\beta-(1 \rightarrow 4)$ Oligosaccharides used are the controlled degradation products of konjac mannan. ${ }^{2}$ $\alpha$ - and $\beta-(1 \rightarrow 6)$ Mannobioses are reversion-products of D-mannose by acid. ${ }^{11}$ The acetyl derivative of the polymer was prepared with acetic anhydride and pyridine in the usual method. ${ }^{12}$ )

Acknowledgement. The authors are grateful to Dr. H. Takeda, Department of General Education, Niigata University, to Drs. T. Sato and S. Suzuki, Research Laboratory of Resources Utilization, Tokyo Institute of Technology, to Dr. Y. Iriki, Department of Education, Shinsyu University, to Dr. C. Kumagai, Research Institute of Brewing, to Dr. K. Ogawa, Sanyo Pulp Co., Ltd., for generous gifts of materials, and to Miss M. Harada for recording the infrared spectra.

\section{REFERENCES}

1) M. L. Wolfrom, M. L. Laver and D. L. Patin, J. Org. Chem., 26, 4535 (1961).

2) K. Kato and K. Matsuda, Agr. Biol. Chem., 33, 1446 (1969); K. Kato, T. Watanabe and K. Matsuda, ibid., 34, 532 (1970).

3) H. Takeda, K. Nishizawa and T. Miwa, "The Science Report of the Tokyo Kyoiku Daigaku," 
Sec. B, Vol. 13 (No. 198), pp. 183 (1968).

4) T. Sato, Nippon Kagaku Zasshi, 88, 982 (1967).

5) Y. Iriki and T. Miwa, Nature, 185, 178 (1960).

6) C. Kumagai, K. Saito and H. Akiyama, Nippon Nogeikagaku Kaishi, 43, 813 (1969).

7) K. Ogawa, K. Matsuda, K. Tamari, M. Yashiro, H. Hirai and S. Kiyooka, Abstracts of Papers, Annual Meeting of the Agricultural Chemical Society of Japan, Sendai, April, 1972, p. 267.

8) E. Maekawa and K. Kitao, Wood Research
(Wood Research Institute, Kyoto University), No. 43, 1 (1968).

9) T. Mizuno, Bull. of Fac. of Agr. Shizuoka Univ., 10, 113 (1960).

10) "Method of Biochemical Analysis," Vol. 3, ed. by $\mathrm{D}$. Glick, Interscience, 1956, p. 213.

11) J. K. N. Jones and W. H. Nicholson, J. Chem. Soc., 1958, 27.

12) J. F. Carson and W. D. Maclay, J. Am. Chem. Soc., 68, 1015 (1946). 